\title{
Semiconductor effective charges from tight-binding theory
}

\author{
J. Bennetto and David Vanderbilt \\ Department of Physics and Astronomy, Rutgers University, Piscataway, New Jersey 08855-0849
}

(October 28, 2018)

\begin{abstract}
We calculate the transverse effective charges of zincblende compound semiconductors using Harrison's tight-binding model to describe the electronic structure. Our results, which are essentially exact within the model, are found to be in much better agreement with experiment than previous perturbation-theory estimates. Efforts to improve the results by using more sophisticated variants of the tight-binding model were actually less successful. The results underline the importance of including quantities that are sensitive to the electronic wavefunctions, such as the effective charges, in the fitting of tight-binding models.
\end{abstract}

77.22.Ej, 77.22.-d, 71.15.Fv

The Born effective charges $e_{T}^{*}$, also known as transverse or dynamic effective charges, are the fundamental quantities which specify the leading coupling between lattice displacements and electrostatic fields in insulators. In general the effective charges are site-dependent tensors,

$$
P_{i}=\sum_{l, j} e_{T_{i j}}^{*(l)} u_{j}^{(l)}+O\left(u^{2}\right),
$$

where $P_{i}$ is the polarization in cartesian direction $i$, and $u_{j}^{(l)}$ is the displacement of sublattice $l$ in cartesian direction $j$. However, for compound semiconductors of the zincblende structure, which are the focus here, it is easily shown that the effective charges are scalars, and are equal and opposite for cation and anion; it is conventional to use the positive cation effective charge to characterize a given compound. The effective charges for a variety of zincblende semiconductors have been computed using $a b$ initio density-functional linear-response theory and have been found to agree very well with experiment. 2.3 However, it is interesting to inquire whether more approximate schemes can give a good accounting of the effective charges in compound semiconductors. If so, additional insight into the chemical and physical factors that affect the $e_{T}^{*}$ might be obtained.

One particularly attractive and well-known approximate scheme is the universal tight-binding model of Harrison 15 It provides a straitforward and computationally efficient approach to calculating electronic properties of solids using a minimal orthogonal $s p^{3}$ basis set, with the Hamiltonian limited to the on-site and nearest neighbor terms. The on-site elements $\epsilon_{s}$ and $\epsilon_{p}$ are taken from calculated free-atom term values, while the interatomic elements $\left(V_{s s \sigma}, V_{s p \sigma}, V_{p p \sigma}\right.$ and $\left.V_{p p \pi}\right)$ are taken to be species-independent "universal" constants times the inverse square of the distance. Given its simplicity, the model is impressively successful in estimating many electronic properties of a wide variety of materials. 45

It is thus natural to ask what the Harrison tightbinding model would predict for the effective charges of the zincblende compound semiconductors. Oddly, this question does not appear to have been answered previously. The only previous work of which we are aware made use of a two-center perturbation approximation to obtain estimates of the effective charges. This approach used an expedient division of the effective charge into "static" and "transfer" charge contributions, with theinterpretation of the latter being open to some question. 1 The purpose of this Report is to present essentially exact calculations of the transverse effective charges computed for zincblende II-VI, III-V, and IVIV semiconductor compounds using the Harrison tightbinding parametrization. While the results could have been obtained using linear-response techniques, we found it simpler to to compute the $e_{T}^{*}$ 's instead from finite differences, calculating directly the change in bulk polarization from a small displacement of one sublattice using the formulation of King-Smith and Vanderbilt. 9 Our calculations are both closer to the experimental values, and more strongly correlated with them, than the previous reults. However, they are still significantly lower than experiment, and the correlation is still not very good. We also tried including off-diagonal position matrix elements, and considered a modified universal tight-binding model that was proposed to incorporate non-orthogonality of the basis functions. 10 Unfortunately, both modifications were found to worsen the results.

The details of our theoretical approach are as follows. We consider each zincblend compound at its experimental lattice constant, with and without displacements of one sublattice along the $\hat{z}$ direction by $\pm 0.0001 \AA$. The Bloch functions are computed in the tight-binding representation using standard direct matrix diagonalization on a mesh of $k$-points. According to the theory of Ref. 9 , the electronic contribution to the polarization takes the form

$$
\mathbf{P}_{e}=-\frac{i e}{(2 \pi)^{3}} \sum_{n=1}^{M} \int_{B Z} d \mathbf{k}\left\langle u_{n \mathbf{k}}\left|\nabla_{\mathbf{k}}\right| u_{n \mathbf{k}}\right\rangle
$$

where the sum runs over occupied bands and the $u_{n \mathbf{k}}$ are the periodic parts of the Bloch wavefuctions, 
TABLE I. The transverse charge $e_{T}^{*}$ for zincblende semiconductors calculated at the experimental lattice spacing $d$, compared with perturbation estimates of Kitamura and Harrison $(\mathrm{KH})$ and experimental values. $\mathrm{O}-$ and $\mathrm{NO}-$ indicate orthogonal and non-orthogonal tight-binding models respectively, while $-\mathrm{O}$ and $-\mathrm{OD}$ refer to diagonal and off-diagonal representations of the position operator.

\begin{tabular}{lrrrrrr}
\hline \hline & $d(\AA)$ & O-D & O-OD & NO-D & KH $^{\text {a }}$ & Expt. $^{\text {b }}$ \\
$\mathrm{SiC}$ & 1.88 & 1.97 & 2.20 & 1.84 & & 2.57 \\
$\mathrm{BN}$ & 1.57 & 1.24 & 0.96 & 1.01 & & 2.47 \\
$\mathrm{BP}$ & 1.97 & -0.09 & -0.18 & -0.23 & & \\
$\mathrm{BAs}$ & 2.07 & -0.39 & -0.42 & -0.54 & & \\
$\mathrm{AlP}$ & 2.36 & 1.92 & 1.61 & 1.64 & & 2.28 \\
$\mathrm{AlAs}$ & 2.43 & 1.75 & 1.50 & 1.50 & & 2.30 \\
$\mathrm{AlSb}$ & 2.66 & 1.48 & 1.22 & 1.32 & & 1.93 \\
$\mathrm{GaP}$ & 2.36 & 1.88 & 1.57 & 1.62 & 0.89 & 2.04 \\
$\mathrm{GaAs}$ & 2.45 & 1.73 & 1.47 & 1.51 & 0.71 & 2.16 \\
$\mathrm{GaSb}$ & 2.65 & 1.41 & 1.12 & 1.29 & 0.40 & 2.15 \\
$\mathrm{InP}$ & 2.54 & 2.26 & 1.94 & 1.99 & 1.26 & 2.55 \\
$\mathrm{InAs}$ & 2.61 & 2.11 & 1.85 & 1.86 & 1.07 & 2.53 \\
$\mathrm{InSb}$ & 2.81 & 1.86 & 2.14 & & 0.75 & 2.42 \\
$\mathrm{BeS}$ & 2.10 & 1.61 & 1.08 & 0.71 & & \\
$\mathrm{BeSe}$ & 2.20 & 1.56 & 1.04 & 0.71 & & \\
$\mathrm{BeTe}$ & 2.40 & 1.51 & 0.96 & 0.57 & & \\
$\mathrm{ZnS}$ & 2.34 & 1.89 & 1.46 & 0.53 & 1.25 & 2.15 \\
$\mathrm{ZnSe}$ & 2.45 & 1.86 & 1.47 & 0.50 & 1.15 & 2.03 \\
$\mathrm{ZnTe}$ & 2.64 & 2.05 & 2.50 & & 0.98 & 2.00 \\
$\mathrm{CdS}$ & 2.53 & 1.98 & 1.61 & 1.10 & & 2.77 \\
$\mathrm{CdTe}$ & 2.81 & 1.92 & 1.53 & 0.41 & 1.24 & 2.35 \\
\hline \hline
\end{tabular}

${ }^{\mathrm{a}}$ Ref. 6 .

${ }^{\mathrm{b}}$ Ref.

$$
u_{n \mathbf{k}}(\mathbf{r})=e^{-i \mathbf{k} \cdot \mathbf{r}} \psi_{n \mathbf{k}}(\mathbf{r})
$$

We are only interested in the $z$ components of $\mathbf{P}$ for the distortions considered. After discretization in $k$-space, these are given 9 as

$$
P_{z}=-\frac{2 e(\Delta k)^{2}}{(2 \pi)^{3}} \sum_{\mathbf{k}_{\perp}} \phi\left(\mathbf{k}_{\perp}\right) .
$$

where $\mathbf{k}_{\perp}=\left(k_{x}, k_{y}\right)$ is discretized on a mesh of spacing $\Delta k$, and the contribution from a string of $J k_{z}$-points takes the Berry-phase formen

$$
\phi\left(\mathbf{k}_{\perp}\right)=\operatorname{Im} \ln \prod_{j=0}^{J-1} \operatorname{det}\left\langle u_{m, \mathbf{k}_{\perp}, k_{j}} \mid u_{n, \mathbf{k}_{\perp}, k_{j+1}}\right\rangle .
$$

Here the argument of the determinant is a $4 \times 4$ matrix corresponding to the fact that $m$ and $n$ run over the four occupied bands. We typically use a discretization onto a $16 \times 16$ mesh in $\mathbf{k}_{\perp}$ space, and extrapolate to $J=\infty$ using strings of $J=32$ and $J=64 k_{z}$ points. The trivial ionic contribution to $P_{z}$ is added, and the value of $e_{T}^{*}$ deduced by simple finite differences.

Strictly speaking, the polarization $\mathbf{P}$ and effective charge $e_{T}^{*}$ are not well-defined until the matrix elements

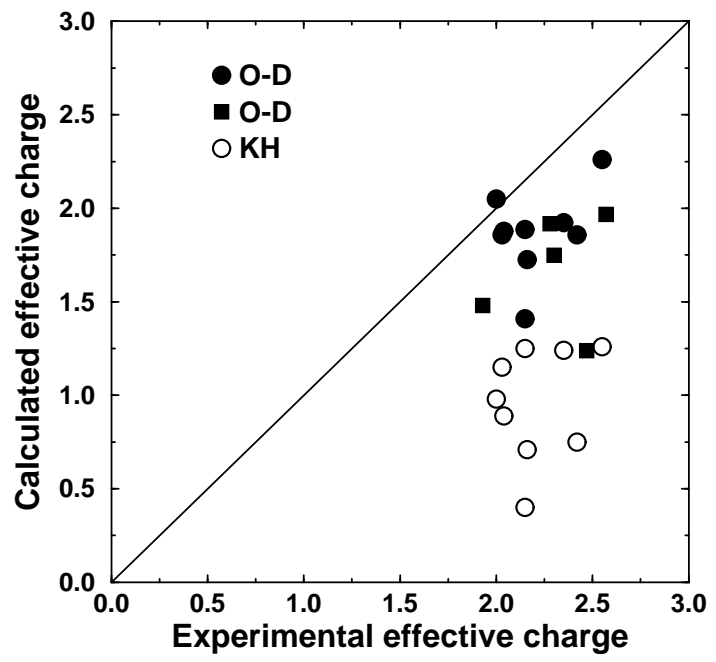

FIG. 1. Comparison of theoretical $e_{T}^{*}$ values from this work (filled symbols) and from perturbation estimates of KH (open symbols, from Ref. 6), plotted against experimental values. Filled squares indicate results for compounds not considered by $\mathrm{KH}$.

of the position operator are specified in the tight-binding basis. In the context of the above formulation, these position matrix elements are needed for the conversion (3) between the $u_{n \mathbf{k}}$ and $\psi_{n \mathbf{k}}$. The simplest ansatz is to assume that the position operator is diagonal in the tightbinding representation, with elements reflecting the coordinates of the atoms. However, such an ansatz is rather unphysical; it would imply that the center of charge of an $s p$ hybrid on an atom would lie exactly at the center of that atom, whereas in reality it would be displaced toward the principal lobe of the hybrid. We report our results first for the simple "diagonal" ansatz. Later, we discuss the effects of trying to improve upon this ansatz, as well as the effect of including the non-orthogonality in the model of Ref. 10 .

The results for the orthogonal Harrison modell using the diagonal representation of $\mathbf{r}$ are given in the column labeled "O-D" (orthogonal, diagonal) for a variety of zincblende structures in Table I.12 The last two columns give the values of the perturbation estimates of Kitamura and Harrison $(\mathrm{KH}), 6$ and the experimental values, for comparison. The data is also represented graphically in Fig. 1. The filled symbols are our results; the open ones are those given in Kitamura and Harrison.6 (The filled squares represent compounds not studied in Ref. 6.) Our calculations shows a clear improvement, although we still systematically underestimate the experimental values of $e_{T}^{*}$. The correlation between our calculations and experiment is not very good, although it should be noted that the lowest filled point is $\mathrm{BN}$, a first row compound for which the model is less accurate.

While the present results are certainly an improvement over the perturbation estimates of $\mathrm{KH}$, there is clearly room for improvement. We thus investigated two possible 


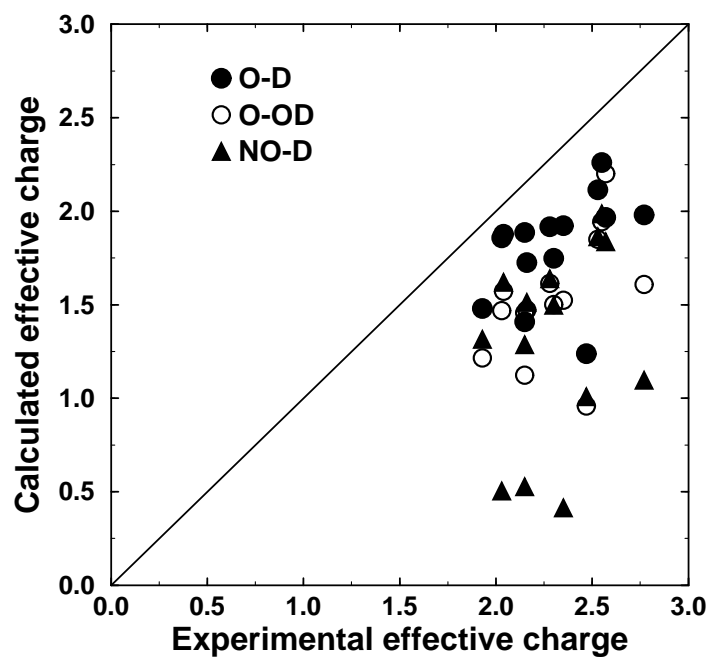

FIG. 2. Comparison of theoretical $e_{T}^{*}$ values using different variants of the tight-binding model, plotted against experimental values. The notation is the same as in Table I.

modifications of the tight-binding model to see whether they would bring the theoretical results into better agreement with experiment. First, we tried going beyond the artificial diagonal ansatz for the tight-binding representation of the position operator by including some offdiagonal terms. Specifically, we included on-site matrix elements between $s$ and $p$ orbitals, e.g., $\left\langle s|z| p_{z}\right\rangle$. The values of these matrix elements were obtained from separate LDA calculations on free (neutral, spin-unpolarized) atoms. By symmetry, off-diagonal $p-p$ matrix elements of $\mathbf{r}$ are zero, and we assumed all off-diagonal intersite elements to be zero as well. The contribution of these extra off-diagonal terms to the polarization $\mathbf{P}$ was calculated as a simple expectation value, using the alreadycalculated wave functions (the Berry-phase approach is not needed). The results are given in the column labeled "O-OD" (orthogonal, off-diagonal) in Table I, and are compared with the previous results (open vs. closed circles) in Fig. 2. Unfortunately, the correction appears to be in the wrong direction, and there is no apparent improvement in the correlation between theoretical and experimental values.

Second, we attempted to improve the results by using a tight-binding model that includes non-orthogonality of the basis, as proposed by van Schilfgaarde and Harrison.10 They used extended Hückel theory to derive the overlap elements

$$
S_{l l^{\prime} m}=\frac{2 V_{l l^{\prime} m}}{K\left(\epsilon_{l}+\epsilon_{l^{\prime}}\right)}
$$

from the original model, $\epsilon_{l}$ and $\epsilon_{l^{\prime}}$ are the onsite energies from the same model, and $K$ is a parameter depending on row of the Periodic Table, chosen to fit the equilibrium spacings of the IV-IV crystals. The Hamiltonian parameters were also renormalized following Eq.
(11) of Ref. 10. Some care is required in the application of the theory of Ref. 9 to this case: the inner product appearing in Eq. (5) has to be generalized to take a form like $\left\langle\phi_{m, \mathbf{k}_{\perp}, k_{j}}\left|S_{\mathbf{k}_{\perp}, \bar{k}}\right| \phi_{n, \mathbf{k}_{\perp}, k_{j+1}}\right\rangle$, where $\left|\phi_{n \mathbf{k}}\right\rangle$ is the vector of tight-binding coefficients corresponding to $\left|u_{n \mathbf{k}}\right\rangle, S_{\mathbf{k}}$ is the overlap matrix at wavevector $\mathbf{k}$, and $\bar{k}=\left(k_{j}+k_{j+1}\right) / 2$. The results are shown in the column labeled "NO-D" (non-orthogonal, diagonal) in Table I, and as the filled triangles in Fig. 2. Once again, this "correction" is seen to act in the wrong direction, worsening the agreement with experiment.

The failures of the above two attempts to improve the tight-binding model are disappointing, but perhaps in hindsight they are not surprising. For the case of the non-orthogonal model, a partial explanation may lie in the fact that the non-orthogonality was added in large part to improve the fit for structures that were not fourfold coordinated, which is not relevant here. But more fundamentally, we note that the model Hamiltonians we tested were developed by fitting to energy bands; thus, the fit included only information about energy eigenvalues, and not the wavefunctions per se. However, the electric polarization is a quantity which depends sensitively on the electronic wavefunctions themselves. Thus, a real improvement in the tight-binding model can probably best be accomplished by including quantities that are sensitive to the wavefunctions, such as $e_{T}^{*}$ values, in the fitting procedure itself.

In summary, we have carried out essentially exact calculations of the transverse effective charge $e_{T}^{*}$ in compound semiconductions within Harrison's universal tightbinding scheme. We find a significantly improved agreement with experiment, compared with previous perturbation estimates. However, the theoretical results still show a systematic underestimate relative to experiment, by an average of $20 \%$. Attempts to improve the agreement by including off-diagonal position matrix elements, or non-orthogonality of the basis, were actually found to lead to a worsening agreement with experiment. Based on this experience, we suggest that it might be helpful to use the effective charge as a fitting parameter in future tight-binding models. Such an approach might lead to a more accurate description of the electronic properties of semiconductors within this class of simple, but very useful, models.

This work was supported by ONR Grant N00014-91-J1184. J.B. acknowledges support of ONR Grant N0001493-I-1097.

${ }^{1}$ R.M. Pick, M.H. Cohen, and R.M. Martin, Phys. Rev. B 1, 910 (1970).

${ }^{2}$ S. de Gironcoli, S. Baroni, and R. Resta, Phys. Rev. Lett. 62, 2853 (1989). 
${ }^{3}$ K. Karch et al., Phys. Rev. B. 50, 17054 (1994).

${ }^{4}$ W.A. Harrison, Phys. Rev. B 24, 5835 (1981).

${ }^{5}$ W.A. Harrison, Electronic Structure and the Properties of Solids (Freeman, San Francisco, 1980) (reprinted by Dover, New York, 1988).

${ }^{6}$ M. Kitamura and W.A. Harrison, Phys. Rev. B 447941 (1991).

${ }^{7}$ M. Kitamura, S. Muramatsu, and W.A. Harrison, Phys. Rev. B 461351 (1992).

${ }^{8}$ In Refs. 8 and 7, the polarization change is taken to have contributions from a "static charge" and a "transfer charge." The former contribution is just the static charge residing on an atom times the distance it moves, while the latter is taken as the induced change in static charge times the interatomic separation. This last approximation is especially questionable; even within the stated assumption that electrons transfer only to neighboring atoms, it neglects the possibility of charge transfer along bonds other than the one on which the perturbation analysis is being performed.

${ }^{9}$ R.D. King-Smith and D. Vanderbilt, Phys. Rev. B 471651 (1993).

${ }^{10}$ M. van Schilfgaarde and W. Harrison, Phys. Rev. B 33 2653 (1986).

${ }^{11}$ M.V. Berry, Proc. Roy. Soc. London A 392451 (1984).

${ }^{12}$ We also calculated the $e_{T}^{*}$ tensor for the same componds in the ideal wurtzite stucture, and found that neither of the two independent $e_{T}^{*}$ components typically differs by more than $\sim 2 \%$ from the zincblende values. 